\title{
Anti-inflammatory Effect of Resveratrol and Polydatin by In Vitro IL-17 Modulation
}

\author{
Giulia Lanzilli, ${ }^{1}$ Andrea Cottarelli, ${ }^{2}$ Giuseppe Nicotera, ${ }^{1}$ Serena Guida, ${ }^{1}$ \\ Giampiero Ravagnan, ${ }^{2}$ and Maria Pia Fuggetta ${ }^{1,3}$
}

\begin{abstract}
Interleukin-17 (IL-17) is a proinflammatory cytokine produced, although not exclusively, by $\mathrm{T}$ helper 17 recently identified as a distinct $\mathrm{T}$ helper lineage mediating tissue inflammation. IL-17 is known to be involved in a number of chronic disorders although the mechanisms regulating its production in inflammatory disease are still unclear. The beneficial properties of the polyphenolic compound resveratrol including its anti-inflammatory, antioxidant, and antitumor effects, its role in the aging process and in the prevention of heart and neurodegenerative diseases are well-known. In addition, derivatives of resveratrol, including glucosylated molecules as polydatin have been linked to similar beneficial effects. We have investigated the effects of resveratrol and polydatin on the in vitro production of IL-17 in a model of inflammation in vitro. The results obtained by activated human peripheral blood mononuclear cells, stimulated with anti-CD3/anti-CD28 monoclonal antibodies and treated with these polyphenolic compounds at different concentrations show that both decrease IL-17 production in a concentration-dependent manner. This study confirms the anti-inflammatory activity of resveratrol and its derivatives and suggests a potential clinical relevance in the therapy of inflammatory diseases.
\end{abstract}

KEY WORDS: resveratrol; polydatin; interleukin 17; inflammation.

\section{INTRODUCTION}

Basic and clinical research provides evidence that inflammatory mechanisms play a central role in the pathogenesis and progression of many pathological conditions.

In particular, chronic inflammatory diseases are characterized by chronic or relapsing immune activation, and there is growing evidence that cytokine deregulation is responsible for the occurrence of these inflammatory and autoimmune pathologies [1-3]. Moreover, inflammation is an important environmental factor that promotes tumorigenesis and progression of established cancerous lesions, and recent studies have started to

Giulia Lanzilli and Andrea Cottarelli contributed equally to this work.

${ }^{1}$ Institute of Translational Pharmacology (IFT), National Council of Research, Rome, Via Fosso del Cavaliere 100, 00173 Rome, Italy

${ }^{2}$ GLURES, Academic SPINOFF, Ca Foscari University of Venice, Venice, Italy

${ }^{3}$ To whom correspondence should be addressed at Institute of Translational Pharmacology (IFT), National Council of Research, Rome, Via Fosso del Cavaliere 100, 00173 Rome, Italy. E-mail: mariapia. fuggetta@ift.cnr.it analyze the mechanisms linking the two pathologies [4]. It is therefore evident that modulation of cytokine production by $\mathrm{T}$ cells can play a fundamental role in the control of various pathologies, and substances able to modulate this production could have important immunopharmacological and therapeutic effects.

The family of $\mathrm{CD} 4+\mathrm{T}$ cells includes different subtypes of T-helper (Th) lymphocytes characterized by specific cytokine profiles: Th1 cells produce interleukin 2 (IL-2), interferon- $\gamma$, and tumor necrosis factor (TNF) and are mainly responsible for cell-mediated immunity, such as retarded hypersensitivity; Th2 cells produce other types of interleukins such as IL-4, IL-5, IL-6, IL-9, IL-10, and IL-13 and are mainly responsible for the regulation of tumor immunity; T helper 17 (Th17) cells, produce IL-17 family, IL-22, IL-21, and CCL20 and have a relevant role in immune response to fungi and extracellular pathogens and inflammatory disorders $[5,6]$. Moreover, Th17 cells have been characterized as potent inducers of tissue inflammation in several autoimmune diseases, such as inflammatory bowel disease, psoriasis, multiple sclerosis, systemic lupus erythematosus, rheu- 
matoid arthritis, and Bechet's disease through the activation of a wide range of inflammatory mediators. The production of IL-17 indeed induces the secretion of chemokines and other cytokines, such as IL-6, IL-8, PGE2, MCP-1, or G-CSF from many kinds of adherent cells like fibroblasts, keratinocytes, epithelial, and endothelial cells [7, 8]. Moreover, the Th17 response has been recently implicated in several models of infection [9].

Evidence from human diseases suggests that, like Th1 and Th2 cells, the in vivo development of Th17 cells from naïve $T$ cells is dependent on antigen presentation by professional antigen presenting cells, co-stimulatory stimulation, and a specific cytokine milieu [10]. In addition, the differentiation factors responsible for their generation have revealed an interesting reciprocal relationship with regulatory $\mathrm{T}\left(\mathrm{T}_{\text {reg }}\right)$ cells, which prevent tissue inflammation and mediate self-tolerance. Furthermore, cytokines that promote Th17 differentiation are distinct from those that promote Th1 and Th2 differentiation. Usually, when the activity of the effector cells is excessive and not balanced by regulatory cells of the immune system, there is the increased risk for development of autoimmune diseases. Therefore, in many autoimmune diseases, the activity of Th17 exceeds that of $\mathrm{T}_{\text {regs }}$ [11-13].

Interleukin-17 is the founding member of a group of cytokines called the IL-17 family. The IL-17 family is comprised of at least six members, including IL-17A, IL17B, IL-17C, IL-17D, IL-17E (IL-25), and IL-17F. Prototype member of the family has been designated IL-17A.

Currently, in addition to Th17 cell subset, a recent literature describes other cells producing IL-17 in response to stress, injury, or pathogens. These innate immune cells have been identified as: $\gamma \delta \mathrm{T}$ cell subset that has an important role at the level of mucosal barrier; iNKT cells, which are activated in response to self and non-self glycolipid antigens presented by the nonpolymorphic MHC class I-like CD1d molecule; and Paneth cells that are highly specialized epithelial cells involved in mucosal homeostasis. They reside in diverse anatomical locations - mainly in the lungs, liver, skin, gut, and secondary lymphoid organs where they have a broad range of immune regulatory functions that has yet to be fully understood [14].

Moreover, IL-17 R mRNA exhibits a broad tissue distribution and has been detected in all cells and tissues tested [15]. Functional studies have provided evidence that IL-17 acts on epithelial cells, endothelial cells, fibroblasts, synoviocytes, and myeloid cells to induce secretion of a variety of mediators including IL-8, CXC ligand (CXCL) 1, CXCL6, IL-6, granulocyte macro- phage colony-stimulating factor, granulocyte colonystimulating factor, TGF- $\beta$, TNF- $\alpha$, and IL- $1 \beta[16,17]$.

Polyphenols, including anthocyanins, flavonoids, and stilbenes, constitute one of the most abundant and ubiquitous group of plant metabolites and are an integral part of the human diet. They are recognized for their beneficial implications in human health such as in the treatment and prevention of cancer and cardiovascular and neurodegenerative diseases. The wide range of biological effects exhibited is generally believed to be the outcome of their powerful antioxidant properties in vitro, which are described in numerous publications [18].

Resveratrol (3,4',5-trihydroxy-trans-stilbene, RES) is a non-flavonoid polyphenol found in several dietary sources, such as grape seeds, berries, peanuts, and red wine.

Resveratrol exists as both the trans- and cis-isomer with numerous reports suggesting trans-resveratrol to be the more stable form in nature and the most bioactive form of this molecule. Trans-resveratrol can be readily converted to cis-resveratrol when exposed to UV light and is unstable when exposed to high $\mathrm{pH}$. In addition to resveratrol isomers, derivatives of resveratrol that include its glucosylated, prenylated, methylated, and hydroxylated modifications have been linked with beneficial activities $[19,20]$.

In particular, polydatin, also known as piceid (resveratrol-3-O- $\beta$-mono-D-glucoside, POLY) is a glucoside of resveratrol in which the glucoside group bonded in position C-3 substitutes a hydroxyl group. This substitution gives rise to conformational changes of the molecule, resulting in changes in the biological properties. Piceid is more resistant to enzymatic oxidation than resveratrol, is soluble in water and unlike resveratrol which penetrates the cell passively it enters the cell via an active mechanism using glucose carriers [21]. This property makes polydatin a molecule endowed with greater bioavailability respect to resveratrol.

Resveratrol is known to have various beneficial effects such as anti-cancer, antioxidative, anti-inflammatory, and cholesterol-lowering activities besides prophylactic effects on cardiovascular diseases. Resveratrol has been shown to suppress proliferation of a wide variety of human tumor cells in vitro which has led to numerous preclinical animal studies to evaluate its cancer preventive and therapeutic potential. Moreover, it has been reported that resveratrol exhibits immunomodulatory properties showing antioxidant and anti-inflammatory activities $[19$, 22, 23]. In particular, Petro TM in its review [24] point out 
the potential therapeutic use of resveratrol, for autoimmune disease through the modulation of Th17 and $\mathrm{T}_{\text {reg }}$ cells.

Similarly to resveratrol, polydatin is able to scavenge free radicals, inhibit platelet aggregation, and oxidize LDL [25, 26].

In this work, we evaluated the effects of different concentrations of RES or POLY on normal human lymphocytes activated in vitro with an inflammatory stimuli. The anti-inflammation outcome of RES and POLY was investigated and compared by evaluating the releasing of proinflammatory IL-17 cytokine in activated T cells.

The evaluation of the immunomodulating effects of RES or POLY was performed using PBLs stimulated via CD3/CD28 molecule activation. This method is currently used to analyze the production of interleukin Il-17 in patients with inflammatory diseases as polymyositis and dermatomyositis, or Crohn's disease and ulcerative colitis [27, 28]. Moreover, this technique, simply and reproducible, permits easy assess to the individual responses in the clinic.

\section{MATERIALS AND METHODS}

\section{Chemicals}

Natural RES and POLY were extracted and kindly supplied by Dr. Fulvio Mattivi, (Fondazione Edmund Mach, Istituto Agrario di San Michele all'Adige (IASMA) Italy). The purity of both compounds tested by HPLC-MS, UV, and NMR was higher than $99 \%$ (method patented and described in) [29, 30].

RES was dissolved in dimethyl sulfoxide (DMSO, Sigma Chemical Co., St. Louis, USA) at $100 \mathrm{mM}$ stock solution, whereas POLY was dissolved in Dulbecco's modified Eagle's medium (DMEM,GIBCO Laboratories, Grand Island, NY, USA) at $100 \mathrm{mM}$. All stock solutions were stored at $-80^{\circ} \mathrm{C}$ and diluted in culture medium just prior to use.

\section{Isolation and Culture of Human Mononuclear Cells}

Buffy coats were obtained from peripheral blood of healthy donors. Peripheral blood mononuclear cells (PBMC) were purified by Ficoll-hypaque gradient centrifugation (Sigma-Aldrich Corp.). The cells at the interface were removed, washed three times by centrifugation at $400 \times \mathrm{g}$ for $10 \mathrm{~min}$ and finally resuspended at the appropriate concentration in RPMI-1640 GLUTAMAX (GIBCO) medium supplemented with $1 \%$ glutamine, $1 \%$ penicillin/streptomycin, and $10 \%$ heat- inactivated $\left(56^{\circ} \mathrm{C}, 30 \mathrm{~min}\right)$ fetal calf serum (Hyclone Laboratories, Logan, UK), herereafter referred to as complete medium (CM).

\section{Treatment of PBMC with RES or POLY and Measurement of Il-17 in Culture Supernatants}

Isolated PBMC, suspended at the concentration of $10^{6}$ cells $/ \mathrm{ml}$ in $\mathrm{CM}$, were treated with $1 \mu \mathrm{g} / \mathrm{ml}$ of antiCD3 mAb (Clone HIT3a, PharMingen International) and incubated at $37^{\circ} \mathrm{C}$ in $5 \% \mathrm{CO} 2$ for 3 days. At the end of incubation, the anti-CD3-stimulated lymphocytes were washed, resuspended in CM containing $1 \mu \mathrm{g} / \mathrm{ml}$ of antiCD28 mAb (clone CD28.2, PharMingen International), and incubated at $37^{\circ} \mathrm{C}$ in $5 \% \mathrm{CO}_{2}$ for additional 3 days.

Immediately before antibody stimulation PBMC were treated with graded concentration $(0.01,0.1,1,2.5$, 5,10 , and $20 \mu \mathrm{g} / \mathrm{ml}$ ) of RES or POLY. As control, the cells were treated with vehicle: DMSO and DMEM, respectively, for RES and POLY treatments.

Cells were analyzed for their cytokine-producing capability on day 6 at the end of treatments. Cell proliferation and viability were determined by the Trypan blue dye exclusion method by manually counting the cells with a hemocytometer. All experiments were carried out in triplicate.

Culture supernatants of cells stimulated with antiCD3/anti-CD28 monoclonal antibodies and treated with different concentrations of RES or POLY were collected for IL-17 detection. The test was carried out by ELISA quantitative sandwich enzyme immunoassay technique (ELISA kit Quantikine, h-IL-17 immunoassay, R\&D Systems, Minneapolis, USA). The detection limit of the assays was approximately $15.0 \mathrm{pg} / \mathrm{ml}$ and is specific for natural and recombinant human IL-17.

\section{Flow Cytometric Analysis}

Cytotoxic effects of RES and POLY treatment were evaluated by flow cytometric analysis. Stimulated and unstimulated lymphocytes, treated with RES and POLY at different concentrations $(2,5,5,10$, and $20 \mu \mathrm{g} / \mathrm{ml})$ and control cells grown only in culture medium, were fixed in $70 \%$ ethanol, washed in PBS, stained with propidium iodide (PI) $50 \mu \mathrm{g} / \mathrm{ml}$ and incubated in presence of $100 \mathrm{KU} /$ $\mathrm{ml}$ of RNAse in the dark at room temperature for $30 \mathrm{~min}$. Cellular fluorescence was measured with a FACscan flow cytometer (Becton Dickinson, Mountain View, CA, USA) using an argon ion laser emitting at $488 \mathrm{~nm}$. Ten thousand events were counted for each sample, and data collection was gated utilizing forward light scatter and side light 
scatter to exclude cell debris and aggregates. Apoptotic cells are represented by a broad hypodiploid peak below the G1 peak (cells with a fractional DNA content) in the red fluorescence channel. This fraction is representative of cells with decreased staining for PI as an indicator for DNA fragmentation associated with apoptotic cell death. All data were recorded using the CellQuest software (Becton \& Dickinson).

\section{IL-17 mRNA Expression-RT-PCR}

Total RNA was extracted from human mononuclear cells using the RNeasy mini kit (Qiagen) according to manufacturer's instructions. The RNA was extracted from: (1) non-activated cells, (2) cells stimulated with anti-CD3/anti-CD28, and (3) cells stimulated with antiCD3/anti-CD28 and treated with $20 \mu \mathrm{g} / \mathrm{ml}$ of POLY. Reverse transcription-PCR was carried out with the PCR CORE kit (Applied Biosystem, Roche) using random hexamers and $1 \mu \mathrm{g}$ total RNA for first-strand synthesis. The cDNA encoding IL-17 was amplified using the oligonucleotides IL-17 forward (5'-ATGACTCCTGGGAAGACCTCATTG-3') and IL-17 reverse (5'ATGACTCCTGGGAAGACCTCA TTG-3') by 40 PCR cycles of $95^{\circ}$ for $30 \mathrm{~s}, 70^{\circ}$ for $30 \mathrm{~s}$, and $72^{\circ}$ for $30 \mathrm{~s}$. As control, the constitutive human $\beta_{2}$ microglobulin cDNA was PCR amplified using the oligonucleotides $\beta_{2}$ forward (5'- GAA TTG CTA TGT GTC TGG GT- 3') and $\beta_{2}$ reverse (5'- CAT CTT CAA ACC TCC ATG ATG- $\left.3^{\prime}\right)$. The amplified products were electrophoresed on $2 \%$ agarose gel in $1 \times$ TAE (Tris acetate EDTA).

\section{Statistical Analysis}

Results are expressed in terms of means $(\mathrm{M}) \pm$ standard error (SE) of the mean. Statistical significance was determined by Student's test analysis.

\section{RESULTS}

\section{Effect of RES and POLY Treatment on IL-17 Production in Activated PBMC In Vitro}

Mononuclear cells isolated from peripheral blood of human healthy donors were treated with RES or POLY at different concentrations $(0.01,0.1,1,2.5,5,10$, and $20 \mu \mathrm{g} / \mathrm{ml}$ ) and activated with monoclonal anti-CD3/ anti-CD28 antibodies. After 6 days, cell samples were evaluated for IL-17 production in culture supernatants by ELISA immunoassay (Fig. 1).<smiles>Oc1ccc(/C=C/c2cc(O)cc(O)c2)cc1</smiles>

\section{Resveratrol}<smiles>OC[C@H]1O[C@@H](Oc2cc(O)cc(/C=C/c3ccc(O)cc3)c2)[C@H](O)[C@@H](O)[C@@H]1O</smiles>

\section{Polydatin}

Fig. 1. Chemical structure of Resveratrol and Polydatin.

The results illustrated in Fig. 2 show that, at higher concentrations, RES and POLY treatments result in a dose-dependent inhibition of IL-17 production by stimulated PBMC. The results show that POLY treatment of activated PBMC induce a marked inhibition IL17 statistically stronger of the inhibition obtained with

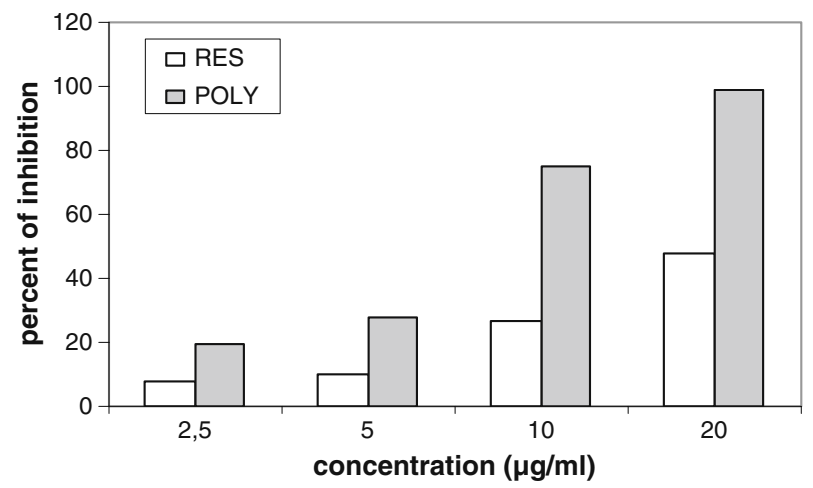

Fig. 2. Effect of RES and POLY on IL-17 production by PBMC activated with anti-CD3/ANTI-CD28 monoclonal antibodies. The results are expressed as percent inhibition of IL-17 production in samples (means of triplicate) treated with different concentrations of RES or POLY respect to untreated stimulated PBMC. The cytokine production was detected by ELISA immunoassay. 
RES at the same concentration. In particular, POLY treatment at $20 \mu \mathrm{g} / \mathrm{ml}$ induces an almost complete inhibition (98\%) whereas RES treatment at $20 \mu \mathrm{g} / \mathrm{ml}$ shows no more than a $50 \%$ of inhibition.

On the other hand, at low concentration $(0.01 \mu \mathrm{g} / \mathrm{ml})$, only RES induces a significant increase of IL-17 produced by stimulated PBMC (Fig. 3a), whereas, POLY treatment at the same concentrations did not show any significant change, although a slight decrease of cytokine production can be observed early, starting from $1 \mu \mathrm{g} / \mathrm{ml}$ (Fig. 3b).
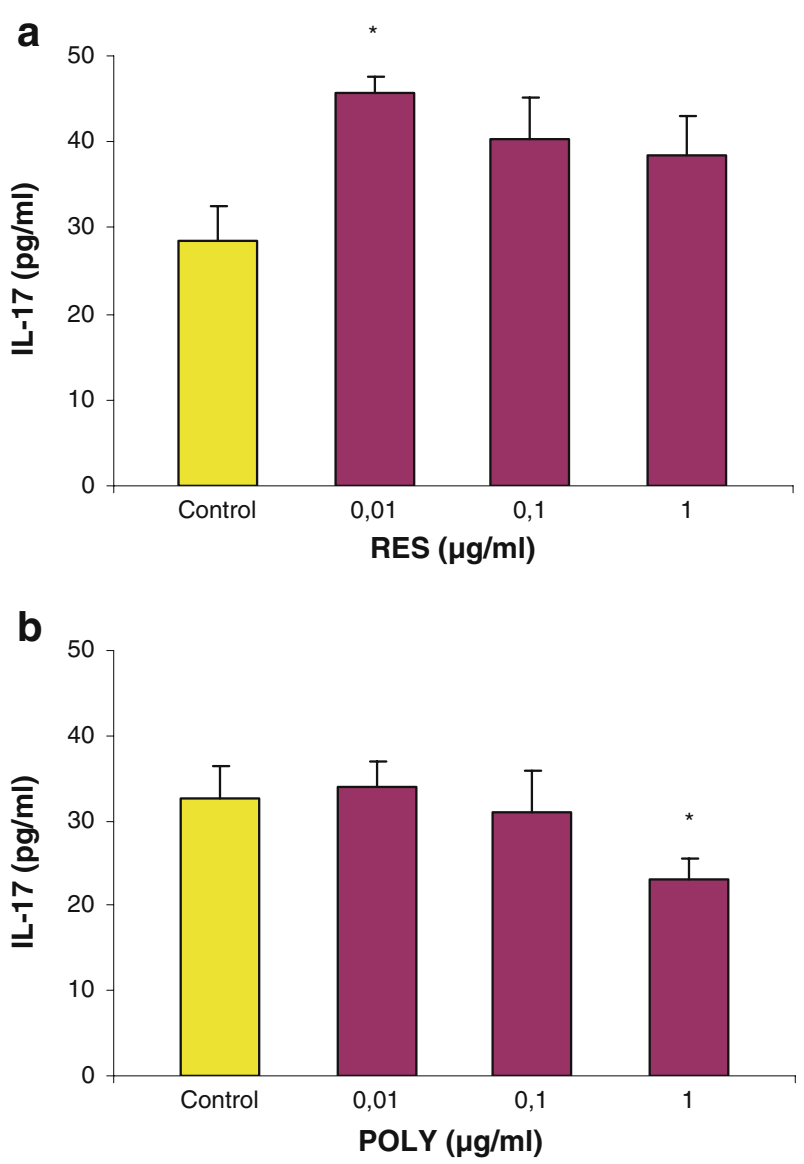

Fig. 3. Effect of low concentrations of RES or POLY on activated lymphocytes. The results are expressed in terms of IL-17 production (picograms per milliliter) in PBMC treated with different low concentrations of RES or POLY respect to untreated control. The Il-17 production was detected by ELISA immunoassay. In panel $\mathbf{a}$, are showed the effects of treatment of PBMC with RES at $0.01,0.1$, and $1 \mu \mathrm{g} / \mathrm{ml}$ concentrations respect to untreated control (only vehicle, DMSO). Bars represent the SE of the mean. ${ }^{*} P<0.01$ (Student's $t$ test). In panel $\mathbf{b}$, are showed the effects of POLY at $0,010,11 \mu \mathrm{g} / \mathrm{ml}$ concentrations respect to untreated control (only vehicle, DMEM) Bars represent the SE of the mean. ${ }^{*} P<0.05$ (Student's $t$ test).

\section{Effect of RES and POLY Treatment on Cytotoxicity of Activated PBMC}

To exclude that, downregulation of IL-17 in culture supernatants was due to cell cytotoxicity by RES or POLY treatment, cultured cells were manually counted to evaluate dead cells by TB exclusion. Data show that cell counts after stimulation and treatment remain substantially unaffected respect to control cells (data not shown).

To evaluate whether RES or POLY treatment induced apoptosis, we tested cultured lymphocytes by flow cytometry analysis. Briefly, unstimulated or stimulated lymphocytes, treated with $2.5,5,10$ e $20 \mu \mathrm{g} / \mathrm{ml}$ of RES or POLY were fixed in acetone/methanol 1:4 in $50 \%$ PBS and labeled with PI $1 \mu \mathrm{g} / \mathrm{ml}$. The results in Fig. 4 show that RES or POLY treatment does not induce a proapoptotic effect both in unstimulated or stimulated cells. Only at $20 \mu \mathrm{g} / \mathrm{ml}$ of POLY treatment a slight significative increase in the number of apoptosis can be observed, both in activated (5.2\%) or unstimulated $(5.9 \%)$ samples.

\section{Effect of RES or POLY on the Expression of IL-17 mRNA in Anti-CD3/ANTI-CD28 Activated Lymphocytes}

Accordingly with data from inhibition of IL-17 activity, IL-17 expression seems to be inhibited by POLY at RNA level as well. IL-17 cDNAs were transcribed and amplified from differently treated human mononuclear cells and results from a representative experiment are shown in Fig. 5. The IL-17 mRNA was clearly down-regulated in cells treated with POLY vs cell-activated and not activated, suggesting its possible regulatory role at transcriptional or post transcriptional level.

\section{DISCUSSION}

IL-17 is a cytokine involved in inflammatory conditions such as autoimmune diseases, psoriasis, arthritis, and inflammatory bowel diseases [10, 11, 13].

The data obtained in this study demonstrate the immunomodulating effects of RES and POLY in an in vitro model of inflammation. The in vitro production of IL-17 by activated PBMC treated with RES or POLY at different concentrations has been evaluated. The results showed that both RES and POLY decrease IL-17 production in a concentration-dependent manner. A strong inhibitory activity (approximately100\%) 

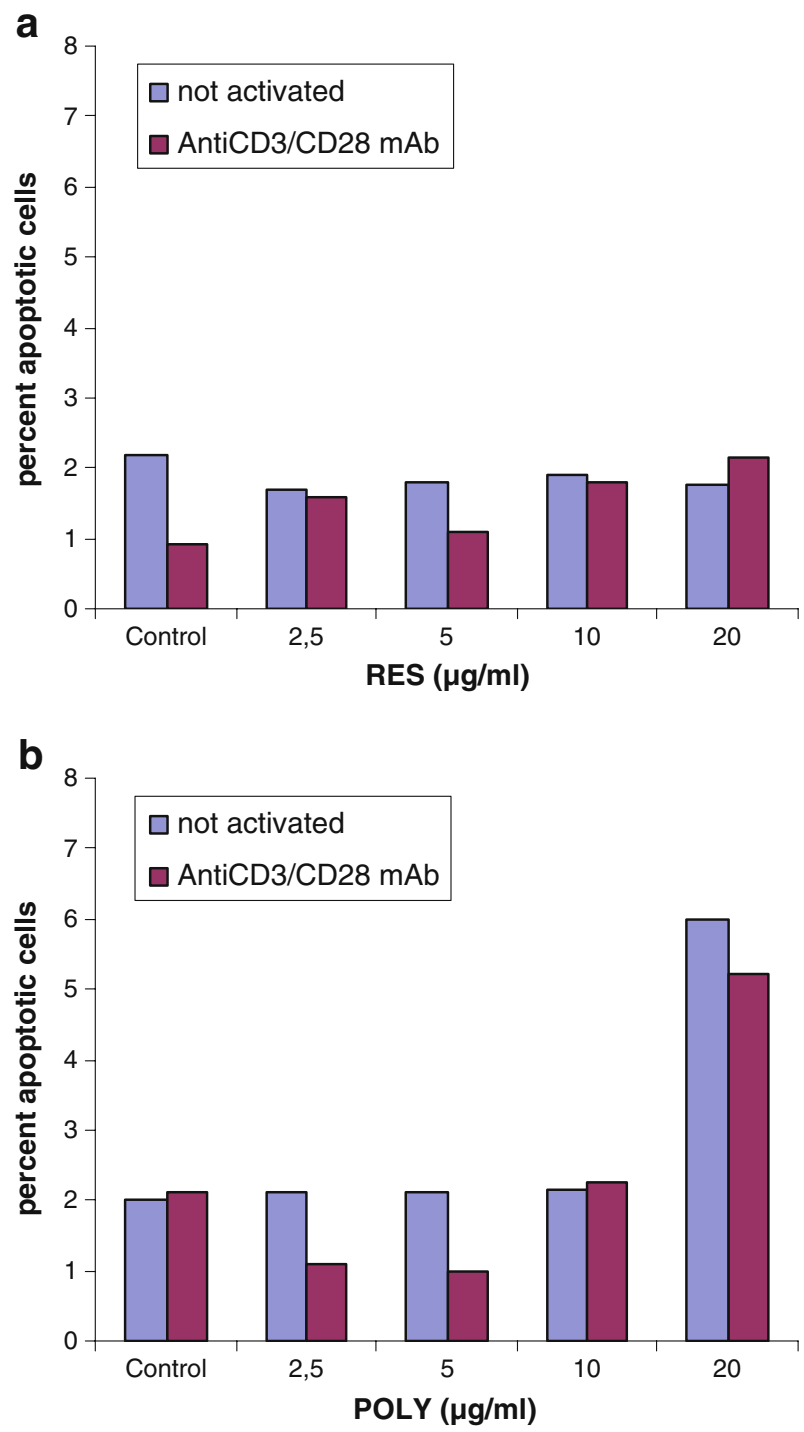

Fig. 4. Effect of RES and POLY on apoptosis induction in PBMC in vitro. Lymphocytes stimulated with antiCD3/antiCD28 mAbs, treated with RES (a) and POLY (b) at 2.5, 5, 10, or $20 \mu \mathrm{g} / \mathrm{ml}$ for 6 days, fixed and labeled with propidium iodide. The percentage of DNA loss was measured by flow cytometry and indicated as the percent of hypodiploid nuclei.

was obtained by POLY treatment at the concentration of $20 \mu \mathrm{g} / \mathrm{ml}$. RES, at the same concentration, also induces a significant inhibition of IL-17 production, although the level of inhibition was lower $(50 \%)$. These results suggest a more effectiveness of POLY in inhibiting IL-17 production. It is well known that in POLY, the hydroxyl group in position C-3 is replaced by a glucoside group that induce a conformational changes of the molecule and resulting in changed biological properties. POLY is more resistant to enzymatic oxidation than RES, it is soluble in water and, unlike RES which penetrates the cell passively, it enters the cell through glucose carriers [21, 25], thus, allowing maximum absorption and a better bioavailability. To establish whether RES and POLY could be active at low concentrations as well, we treated stimulated PBMC with either RES or POLY at 0.01 , 0.1 , and $1 \mu \mathrm{g} / \mathrm{ml}$. The results showed that only RES at $0.01 \mu \mathrm{g} / \mathrm{ml}$ increases the IL-17 production. This finding is in agreement with our previous studies on immunomodulating activity of RES describing the effects on cell function and cytokine production in three models of in vitro immune response [31]. We previously demonstrated that in vitro treatment of immune cells with RES induces a biphasic effect: immunostimulation at low concentrations and strong inhibition at higher concentrations, for natural (NK cell) activity, antigen-specific immune responses and cytokine production. Son TG et al. successively confirmed this biphasic dose-response effect on different cells and pointed out the hormetic effect by several phytochemicals compounds [32].

RES is reported as a potent inducer of apoptosis $[33,34]$. To exclude the possibility that a diminished amount of IL-17 after RES or POLY treatment was

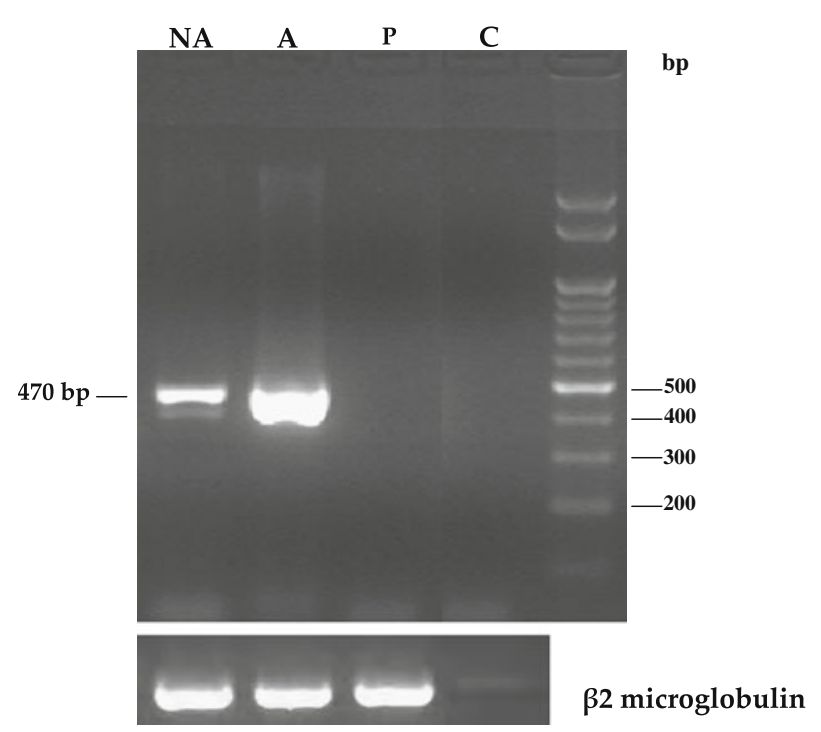

Fig. 5. Effect of poly on IL-17 transcription on treated and not treated PBMC. RT-PCR from untreated PBMC (lane $N A$ ), antiCD3/antiCD28 mAbs stimulated PBMC (lane $A$ ), and PBMC stimulated and treated with POLY at $20 \mu \mathrm{g} / \mathrm{ml}$ (lane $P$ ). $C$ negative control. 
related to a reduction of number of viable cells, we tested the effect of RES or POLY treatment on apoptosis induction. Flow cytometry analysis evidenced that only highest concentration of POLY induced a modest proapoptotic effect. These results are also in agreement with data from viable cell enumeration by TB exclusion (data not shown) and confirm that activated lymphocytes are more resistant to apoptotic cell death [35].

To further investigate the effect of POLY at the highest concentration $(20 \mu \mathrm{g} / \mathrm{ml})$, we studied the IL-17 gene expression at the molecular level in stimulated or unstimulated lymphocytes in vitro.

Accordingly, with data obtained about the IL-17 inhibition in supernatants, IL-17 expression seems to be inhibited in POLY-treated PBMC at RNA level. From preliminary data (Fig. 5), the IL-17 mRNA was clearly down-regulated in activated cells treated with POLY, suggesting its possible regulatory role at transcriptional or post-transcriptional level.

As previously described, uncommitted $\mathrm{T}$ helper cells can be induced to differentiate to specific lineages according to the local cytokine milieu, towards Th1, Th2, Th17, and $\mathrm{T}_{\text {reg }}$ phenotypes in a mutually exclusive manner [5-7]. Each phenotype is characterized by unique signaling pathway and expression of specific transcription factors, notably T-bet for Th1, GATA-3 for Th2, forkhead box P3 (FoxP3) for $\mathrm{T}_{\text {regs }}$ and receptorrelated orphan receptor (ROR)alpha and RORgamma for Th17 cells. [11, 13, 36].

Overexpression of ROR $\gamma \mathrm{t}$ promotes Th17 differentiation; conversely, ROR $\gamma \mathrm{t}$ deficiency results in profound Th17 deficiency. However, ROR $\gamma \mathrm{t}$ defect did not completely abolish Th17 differentiation. Th17 cells also express another orphan nuclear receptor $\operatorname{ROR} \alpha$, which is induced by TGF- $\beta$ and IL- 6 in a STAT3dependent manner. Overexpression of ROR $\alpha$ promotes Th17 differentiation and significantly upregulates IL-17 expression. ROR $\alpha$ deficiency results in reduced IL-17 expression in vitro and in vivo. Furthermore, $\operatorname{ROR} \alpha$ and ROR $\gamma \mathrm{t}$ co-expression synergistically drive greater Th17 differentiation especially under non-favorable conditions $[13,37,38]$.

Signal transducer and activator of transcription 3 (STAT3) acts as an important mediator in multiple biological processes induced by different cytokines [39]. ROR $\gamma \mathrm{t}$ the first transcription factor to be selectively expressed in Th17 cells is regulated by STAT3 [40]. Overexpression of a hyperactive STAT3 enhanced Th17 differentiation while STAT3 deficiency impairs Th17 differentiation in vitro $[38,39]$ and in vivo [41]. STAT3 has been shown to bind to IL-17 gene promoter [42] and appears to control IL-17 gene expression [43]. The precise biochemical function of STAT3 is unclear, but it could be supposed that it affects the regulation of lineage-specific master transcription factors expression. Moreover, recently, IL-21 was reported to regulate Th17 differentiation, as an autocrine factor induced by IL-6 [44]. STAT3 is also needed for IL-6 induction and is required for IL-21mediated Th17 differentiation [41].

Resveratrol attenuates the activation of immune cells and modulates the subsequent synthesis and release of inflammatory mediators through the inhibition of transcriptional factors such as nuclear factor-kappaB [45]. Resveratrol has also been shown to control other various transcription factors as STAT3, HIF-1alpha, beta-catenin, and PPAR-gamma [46]. Resveratrol could significantly inhibit the JAK1/STAT3 signal transduction pathway, through down-regulation of pJAK1 and pSTAT3 expression and reducing the phosphorylation of JAK1 and STAT3 in a dose- and time-dependent manner [47].

It is reasonable to hypothesize that both RES and POLY, reducing the phosphorylation of STAT3, regulate $\mathrm{ROR} \gamma \mathrm{t}$, the first transcription factor selectively expressed in Th17 cells, and consequentially induce a reduction of IL-17 production. Further research is in progress in our laboratory to investigate this molecular pathway.

\section{ACKNOWLEDGMENTS}

This work was partially supported by Ministero dell'Istruzione, dell'Università e della Ricerca Rome, Italy.

We are grateful to Fulvio Mattivi for generously providing purified resveratrol and polydatin.

\section{REFERENCES}

1. Kopf, M., M.F. Bachmann, and B.J. Marsland. 2010. Averting inflammation by targeting the cytokine environment. Nature Reviews. Drug Discovery 9(9): 703-718.

2. Valledor, A.F., M. Comalada, L.F. Santamaría-Babi, J. Lloberas, and A. Celada. 2010. Macrophage proinflammatory activation and deactivation a question of balance. Advances in Immunology 108: $1-20$.

3. Duarte, J., A. Agua-Doce, V.G. Oliveira, J.E. Fonseca, and L. Graca. 2010. Inflammatory bowel disease (Proinflammatory cytokines underlying the inflammation of Crohn's disease. PLoS ONE 5(5): e10558. 
4. Reuter, S., S.C. Gupta, M.M. Chaturvedi, and B.B. Aggarwal. 2010. Oxidative stress, inflammation and cancer how are they linked? Free Radical Biology \& Medicine 49(11): 1603-1616. Epub 2010 Sep 16.

5. Zhu, J., and W.E. Paul. 2010. Heterogeneity and plasticity of T helper cells. Cell Research 20(1): 4-12. Epub 2009 Dec 15.

6. Wan, Y.Y. 2010. Multi-tasking of helper T cells. Immunology 130 (2): $166-171$.

7. Noma, T. 2010. Helper T cell paradigm: Th17 and regulatory $T$ cells involved in autoimmune inflammatory disorders, pathogen defense and allergic diseases. Nihon Rinsho Men'eki Gakkai Kaishi 33(5): 262-271.

8. Chen, S., T.R. Crother, and M. Arditi. 2010. Emerging role of IL17 in atherosclerosis. Journal of Innate Immunity 2(4): 325-333. Epub 2010 May 7. Review.

9. Netea, M.G., and L. Maródi. 2010. Innate immune mechanisms for recognition and uptake of Candida species. Trends in Immunology 31(9): 346-353. Epub 2010 Aug 11. Review.

10. Leung, S., X. Liu, L. Fang, X. Chen, T. Guo, and J. Zhang. 2010. The cytokine milieu in the interplay of pathogenic Th1/Th17 cells and regulatory $\mathrm{T}$ cells in autoimmune disease. Cellular \& Molecular Immunology 7(3): 182-189. Epub 2010 Apr 12. Review.

11. Mai, J., H. Wang, and X.F. Yang. 2010. Th 17 cells interplay with Foxp3+ Tregs in regulation of inflammation and autoimmunity. Frontiers in Bioscience 15: 986-1006. Review.

12. Jäger, A., and V.K. Kuchroo. 2010. Effector and regulatory T-cell subsets in autoimmunity and tissue inflammation. Scandinavian Journal of Immunology 72(3): 173-184. Review.

13. Afzali, B., P. Mitchell, R.I. Lechler, S. John, and G. Lombardi. 2010. Translational mini-review series on Th17 cells: induction of interleukin-17 production by regulatory $\mathrm{T}$ cells. Clinical and Experimental Immunology 159(2): 120-130. Epub 2009 Nov 11.

14. Cua, D.J., and C.M. Tato. 2010. Innate IL-17-producing cells: the sentinels of the immune system. Nature Reviews. Immunology 10 (7): 479-489. Epub 2010 Jun 18. Review. Erratum in: Nat Rev Immunol. 2010 Jul;10(7):following 489. Nat Rev Immunol. 2010 Aug;10(8):611.

15. Moseley, T.A., D.R. Haudenschild, L. Rose, and A.H. Reddi. 2003. Interleukin-17 family and IL-17 receptors. Cytokine \& Growth Factor Reviews 14(2): 155-174.

16. Cianci, R., D. Pagliari, V. Pietroni, R. Landolfi, and F. Pandolfi. 2010. Tissue infiltrating lymphocytes: the role of cytokines in their growth and differentiation. Journal of Biological Regulators and Homeostatic Agents 24(3): 239-249.

17. Guglani, L., and S.A. Khader. 2010. Th17 cytokines in mucosal immunity and inflammation. Curr Opin HIV AIDS 5(2): 120-127.

18. Sies, H. 2010. Polyphenols and health: update and perspectives. Archives of Biochemistry and Biophysics 501(1): 2-5. Epub 2010 Apr 14. Review.

19. Shakibaei, M., K.B. Harikumar, and B.B. Aggarwal. 2009. Resveratrol addiction: to die or not to die. Molecular Nutrition \& Food Research 53(1): 115-128. Review.

20. Cottart, C.H., V. Nivet-Antoine, C. Laguillier-Morizot, and J.L. Beaudeux. 2010. Resveratrol bioavailability and toxicity in humans. Molecular Nutrition \& Food Research 54(1): 7-16.

21. Fabris, S., F. Momo, G. Ravagnan, and R. Stevanato. 2008. Antioxidant properties of resveratrol and piceid on lipid peroxidation in micelles and monolamellar liposomes. Biophysical Chemistry 135(1-3): 76-83.

22. Bertelli, A.A., and D.K. Das. 2009. Grapes, wines, resveratrol, and heart health. Journal of Cardiovascular Pharmacology 54(6): 468476. Review.

23. Gullett, N.P., A.R. Ruhul Amin, S. Bayraktar, J.M. Pezzuto, D.M. Shin, F.R. Khuri, B.B. Aggarwal, Y.J. Surh, and O. Kucuk. 2010.
Cancer prevention with natural compounds. Seminars in Oncology 37(3): 258-281. Review.

24. Petro TM. 2010. Regulatory role of resveratrol on Th17 in autoimmune disease. Int Immunopharmacol. Aug 12.

25. Mikulski, D., and M. Molski. 2010. Quantitative structureantioxidant activity relationship of trans-resveratrol oligomers, trans-4, 4'-dihydroxystilbene dimer, trans-resveratrol-3-O-glucuronide, glucosides: trans-piceid, cis-piceid, trans-astringin and transresveratrol-4'-O-beta-D-glucopyranoside. European Journal of Medicinal Chemistry 45(6): 2366-2380.

26. Du, J., L.N. Sun, W.W. Xing, B.K. Huang, M. Jia, J.Z. Wu, H. Zhang, and L.P. Qin. 2009. Lipid-lowering effects of polydatin from Polygonum cuspidatum in hyperlipidemic hamsters. Phytomedicine 16(6-7): 652-658.

27. Rovedatti, L., T. Kudo, P. Biancheri, M. Sarra, C.H. Knowles, D.S. Rampton, G.R. Corazza, G. Monteleone, A. Di Sabatino, and T.T. Macdonald. 2009. Differential regulation of interleukin 17 and interferon gamma production in inflammatory bowel disease. Gut 58(12): 1629-1636.

28. Shen H, Xia L, Lu J, Xiao W. 2011. Interleukin-17 and interleukin23 in patients with polymyositis and dermatomyositis. Scand J Rheumatol. Jan 13.

29. Mattivi, F., F. Reniero, and S. Korhammeret. 1995. Isolation, characterization, and evolution in red wine vinification of resveratrol monomers. Journal of Agricultural and Food Chemistry 43: 1620-1823.

30. G. Ravagnan, R. Falchetti, G. Lanzilli, M.P. Fuggetta, M. Tricarico and F. Mattivi. Solid phase extraction of trans-resveratrol from wines for HPLC analysis. Use of extracts from spermatophyte plants with immunomodulating activity, Pat. EP 1292 320)

31. Falchetti, R., M.P. Fuggetta, G. Lanzilli, M. Tricarico, and G. Ravagnan. 2001. Effects of resveratrol on human immune cell function. Life Sciences 70(1): 81-96.

32. Son, T.G., S. Camandola, and M.P. Mattson. 2008. Hormetic dietary phytochemicals. Neuromolecular Medicine 10(4): 236-246. Epub 2008 Jun 10. Review.

33. Fuggetta, M.P., G. Lanzilli, M. Tricarico, A. Cottarelli, R. Falchetti, G. Ravagnan, and E. Bonmassar. 2006. Effect of resveratrol on proliferation and telomerase activity of human colon cancer cells in vitro. Journal of Experimental \& Clinical Cancer Research 25(2): 189.

34. Athar, M., J.H. Back, L. Kopelovich, D.R. Bickers, and A.L. Kim. 2009. Multiple molecular targets of resveratrol: anti-carcinogenic mechanisms. Archives of Biochemistry and Biophysics 486(2): 95102. Review.

35. Shahabuddin, S. 1993. Expression of IL-2 receptor subunit p55 (CD25) on normal human lymphocytes. Clinical Immunology and Immunopathology 69(2): 175-179.

36. Sundrud, M.S., and M.A. Nolan. 2010. Synergistic and combinatorial control of $\mathrm{T}$ cell activation and differentiation by transcription factors. Current Opinion in Immunology 22(3): 286-292.

37. Burgler, S., P.Y. Mantel, C. Bassin, N. Ouaked, C.A. Akdis, and C. B. Schmidt-Weber. 2010. RORC2 is involved in T cell polarization through interaction with the FOXP3 promoter. Journal of Immunology 184(11): 6161-6169.

38. Ivanov, I.I., B.S. McKenzie, L. Zhou, C.E. Tadokoro, A. Lepelley, J.J. Lafaille, D.J. Cua, and D.R. Littman. 2006. The orphan nuclear receptor RORgammat directs the differentiation program of proinflammatory IL-17+ T helper cells. Cell 126(6): 1121-1133.

39. Jarnicki, A., T. Putoczki, and M. Ernst. 2010. Stat3: linking inflammation to epithelial cancer-more than a "gut" feeling? Cell Division 5: 14.

40. Wei, L., A. Laurence, K.M. Elias, and J.J. O'Shea. 2007. IL-21 is produced by Th17 cells and drives IL-17 production in a STAT3dependent manner. The Journal of Biological Chemistry 282(48): 34605-34610. 
41. Yang, X.O., A.D. Panopoulos, R. Nurieva, S.H. Chang, D. Wang, S.S. Watowich, and C. Dong. 2007. STAT3 regulates cytokinemediated generation of inflammatory helper T cells. The Journal of Biological Chemistry 282(13): 9358-9363.

42. Ivanov, I.I., L. Zhou, and D.R. Littman. 2007. Transcriptional regulation of Th17 cell differentiation. Seminars in Immunology 19 (6): 409-417.

43. Chen, Z., A. Laurence, Y. Kanno, M. Pacher-Zavisin, B.M. Zhu, C. Tato, A. Yoshimura, L. Hennighausen, and J.J. Shea. 2006. Selective regulatory function of Socs 3 in the formation of IL-17secreting T cells. Proceedings of the National Academy of Sciences of the United States of America 103(21): 8137-8142.
44. Korn, T., E. Bettelli, W. Gao, A. Awasthi, A. Jäger, T.B. Strom, M. Oukka, and V.K. Kuchroo. 2007. IL-21 initiates an alternative pathway to induce proinflammatory $\mathrm{T}(\mathrm{H}) 17$ cells. Nature 448(7152): 484- 487.

45. Das, S., and D.K. Das. 2007. Anti-inflammatory responses of resveratrol. Inflammation \& Allergy Drug Targets 6(3): 168-173. Review.

46. Harikumar, K.B., and B.B. Aggarwal. 2008. Resveratrol: a multitargeted agent for age-associated chronic diseases. Cell Cycle 7(8): $1020-1035$.

47. Li, T., W. Wang, H. Chen, T. Li, and L. Ye. 2010. Evaluation of anti-leukemia effect of resveratrol by modulating STAT3 signaling. International Immunopharmacology 10(1): 18-25. 